\title{
Built-in Self Test of High Speed Analog-to-Digital Converters
}

\author{
Edinei Santin, Luis B. Oliveira, and Joao Goes
}

Signals found in nature need to be converted to the digital domain through analog-to-digital converters (ADCs) to be processed by digital means [1]. For applications in communication and measurement [2], [3] high conversion rates are required. With advances of the CMOS technology, the conversion rates of CMOS ADCs are now well beyond the gigasamples per second (GS/s) range, but only moderate resolutions are required [4]. These ADCs need to be tested after fabrication and, if possible, during field operation. The test costs are a very significant fraction of their production cost [5]. This is mainly due to lengthy use of very expensive automated test equipment (ATE) to apply specific test stimuli to the devices under test (DUT), and to collect and analyse their responses.

With high conversion rates it is difficult to interface the ADCs to the test instruments due: extra parasitics, line trace mismatches, interferences within the printed circuit board (PCB), and the instrument test probes. This becomes even more challenging in the case of systems-on-a-chip, system-in-a-package, and three-dimensional (3D) chips, where the accessibility of the ADCs for testing purposes is restricted [6].

In this paper we discuss the use of built-in self-test (BIST) techniques for testing high speed ADCs and we present a specific solution, in which the analog input signal and the clock are generated internally.

\section{BIST for High Speed ADCs}

Using advanced CMOS technologies, the cost per transistor decreases and integrated solutions become competitive. Thus, more specific functionalities can be fully integrated on-chip. Moreover, the reliability of the manufacturing process maintains high, allowing reliable function to be implemented on-chip as well.

The implementation of test-related functions directly on-chip during the IC design phase, which is called design for testability, evolved firstly for purely digital ICs and then migrated to mixed-signal devices. Using a BIST approach contributes to additional area, increases design time and effort, and may degrade the performance of the DUT due to intrusive test structures. Table 1 summarizes the pros and cons of a BIST solution. 
Table 1 - Summary of advantages and disadvantages of BIST.

\begin{tabular}{|c|c|}
\hline Advantages & Disadvantages \\
\hline \hline vertical testability (wafer to system) & area overhead \\
\hline high diagnosis resolution & performance penalties \\
\hline full-speed testing & additional design time \& effort \\
\hline reduced need for external test equipment & additional risk to project \\
\hline reduced test development time \& effort & difficulty to reach full accuracy \\
\hline reduced manufacturing test time \& effort & \\
\hline reduced time-to-market & \\
\hline
\end{tabular}

As discussed above, as a result of the increase of the conversion rate of ADCs, from the present few $\mathrm{GS} / \mathrm{s}$, their testing becomes progressively more time-consuming and costly. Here we present a novel solution, which consists of using two small area synchronized phase-locked loops (PLLs) to generate high frequency sinusoidal and clock signals. We analyse the possibility of using a dedicated BIST approach for testing high speed, moderate resolution ADCs, either embedded into complex systems or as standalone components. This BIST approach is based on two compact and easy-to-integrate oscillators that generate high frequency test stimuli (analog input and clock) to the DUT. Instead of using free-running oscillators for generating the analog input and clock test stimuli, we propose to employ two phase-locked-loops to improve the phase noise of the generated signals and, more importantly, to allow coherent sampling. The use of coherent sampling is beneficial since it simplifies the processing of the output data and a coherent sampled signal leads to a coherent spectrum, which avoids undesirable artefacts due to spectral leakage. This fully integrated in CMOS technology BIST approach, which reduces test costs by avoiding the need of expensive mixed-signal ATEs, improves reliability by avoiding interfacing critical signals between the DUT and ATE, and enhances feasibility by carrying out the test procedure completely on-chip, is the main contribution of this paper. Although the DUT used in this work employs a pipelined ADC topology, the approach can be applied to other converter topologies, since it "sees" the DUT as an almost ideal "black box". The conceptual overview of the proposed approach is shown in Fig. 1. 

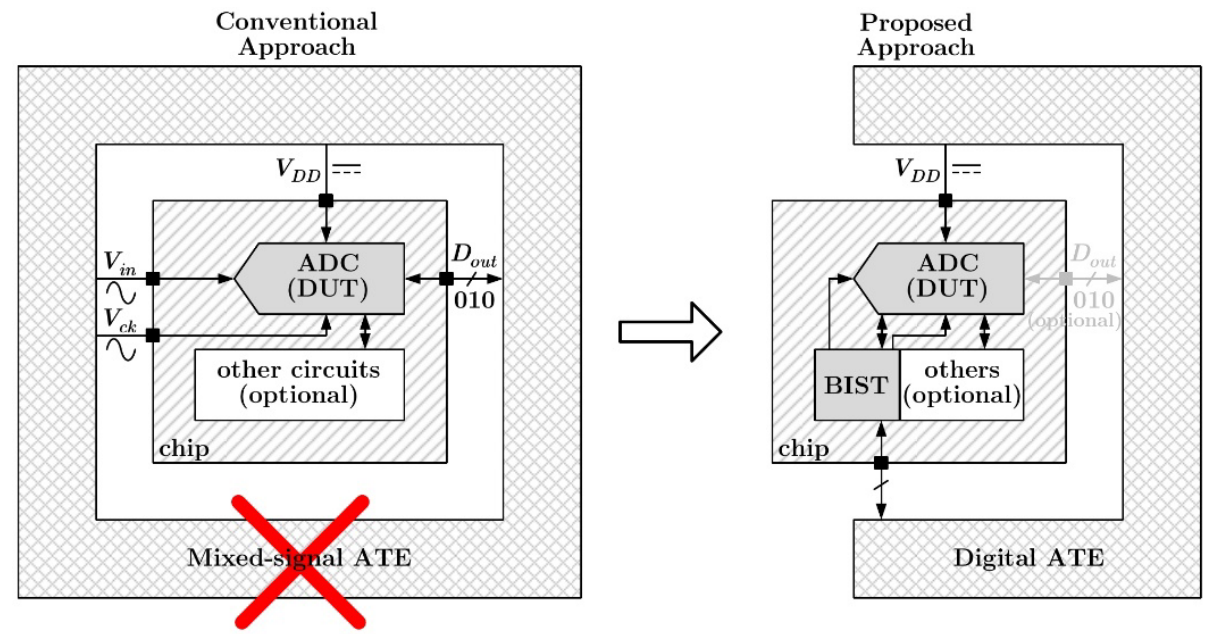

Fig. 1. Overview of the proposed idea.

Using the proposed BIST approach, the only off-chip component required is a low frequency and stable reference signal. Digital signal processing (DSP) techniques for output response analysis are used, which rely primarily on spectral computations using fast Fourier transform (FFT) algorithms. The proposed concept was implemented in an integrated circuit featuring two PLLs for test stimuli generation and an 8-bit $500 \mathrm{MS} / \mathrm{s} \mathrm{ADC}$ as the DUT and was validated with silicon measurements. In a $0.13 \mu \mathrm{m}$ CMOS technology, the area overhead introduced by the two PLLs is only $0.052 \mathrm{~mm}^{2}$.

\section{PROPOSED ADC BIST ARCHITECTURE}

The ADC BIST architecture proposed in this work is illustrated in Fig. 2. It is composed of two integer$N$ PLLs (PLLIN and PLLCK) that share the same external reference clock to ensure signal synchronization. This synchronization is necessary to achieve coherent sampling. PLLIN generates low distortion, high frequency, and low jitter sine wave signals, while PLLCK generates high frequency and low jitter square wave clock signals, which are used to drive the ADC during the test procedure. The only off-chip component required is a stable and low frequency reference clock, which may be implemented, for example, by an inexpensive crystal oscillator. The digitized samples are processed by a DSP (e.g. to execute a FFT algorithm), in order to calculate the most important functional performances of the DUT. The results may then be compared, for example, with different effective resolution thresholds (e.g. 6, 7, and 8 bits) up to the maximum resolution allowed by the self-testing system and, for each comparison, a pass or fail indication shown. With this, ADCs having different resolutions are sorted and it becomes possible to check the performance degradation during the ADC life time. A test controller takes care of the test application and sequence. 


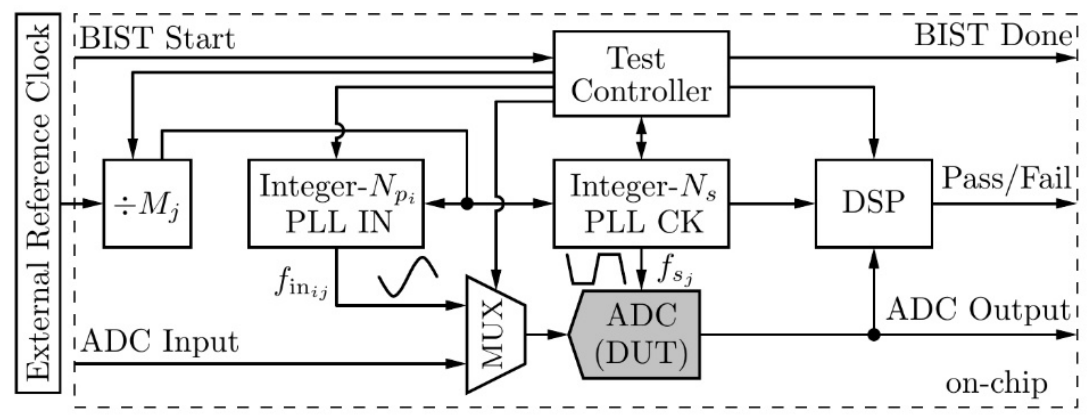

Fig. 2. Proposed BIST architecture for coherent testing of high speed ADCs.

When the two PLLs are locked, the input and sampling frequencies of the ADC are, respectively,

$$
\begin{aligned}
& f_{\mathrm{in}_{i j}}=N_{p_{i}} \frac{f_{\mathrm{ref}}}{M_{j}} \\
& f_{s_{j}}=N_{s} \frac{f_{\mathrm{ref}}}{M_{j}}
\end{aligned}
$$

where $f_{\text {ref }}$ is a stable external reference frequency, $\underline{N}_{\underline{s}}$ is the number of collected samples, and $M_{j}$ and $N_{p i}$ $(i, j=1,2, \ldots)$ are integer programmable frequency divider values, which enable different frequencies for the input and sampling signals. From Eqs. (1) and (2) coherent sampling is achieved (i.e. $f_{\text {in }} / f_{s}=N_{p} / N_{s}$ ).

The architecture parameters $N_{s}, N_{p i}$, and $M_{j}$ are selected according to the resolution of the ADC under test, the external reference frequency, the desired sampling frequencies $\left(\hat{f}_{s j}\right)$, and the desired normalized input frequencies $\left(\hat{f}_{\text {ini }} / f_{s}\right)$. $N_{s}$ is a power of two ideally given by $N_{s}>\pi 2^{N} \approx 2^{N+2}[7]$, where $N$ is the number of bits of the ADC. $N_{p i}(i=1,2, \ldots)$ is given by

$$
N_{p_{i}}=\left\{\frac{\hat{f}_{\mathrm{in}_{i}}}{f_{s}} N_{s}\right\}
$$

where $\{x\}$ means the nearest odd integer value of $x$. Note that odd integer values for $N_{p i}$ ensure irreducibility with respect to $N_{s}$, since the latter is a power of two (this irreducible ratio is another requisite of coherent sampling). $M_{j}(j=1,2, \ldots)$ is given by

$$
M_{j}=\left\langle\frac{f_{\mathrm{ref}} N_{s}}{\hat{f}_{j}}\right\rangle
$$

where $\langle x\rangle$ is the nearest integer value of $x$. As an example, Table 2 gives the architecture parameters and the resulting frequency map for a hypothetical 6-bit $\mathrm{ADC}$ with $f_{\mathrm{ref}}=4 \mathrm{MHz}, \hat{f}_{s j}=250,500$, and $1000 \mathrm{MS} / \mathrm{s}$, 
and $f_{\text {ini }} / f_{s}=1 / 2,1 / 4$, and $1 / 8$. We can see that the actual analog input and sampling frequencies are slightly different from the desired ones, but this is required to guarantee coherent sampling.

Table 2 - Programmable parameters and frequency map for a 6-bit ADC.

\begin{tabular}{|l|l|lll|}
\hline \multicolumn{2}{|l|}{$f_{\text {ref }} \equiv 4 \mathrm{MHz}$} & $\hat{f}_{s_{1}} \equiv 250$ & $\hat{f}_{s_{2}} \equiv 500$ & $\hat{f}_{s_{3}} \equiv 1000$ \\
\hline \multirow{2}{*}{$N \equiv 6$ bits } & \multirow{2}{*}{$N_{s}=256$} & $M_{1}=4$ & $M_{2}=2$ & $M_{3}=1$ \\
\cline { 3 - 5 } & & $f_{s_{1}}=256$ & $f_{s_{2}}=512$ & $f_{s_{3}}=1024$ \\
\hline$\hat{f}_{\text {in }_{1}} / f_{s} \equiv 1 / 2$ & $N_{p_{1}}=127$ & $f_{\text {in }_{11}}=127$ & $f_{\mathrm{in}_{12}}=254$ & $f_{\mathrm{in}_{13}}=508$ \\
\hline$\hat{f}_{\mathrm{in}_{2}} / f_{s} \equiv 1 / 4$ & $N_{p_{2}}=63$ & $f_{\mathrm{in}_{21}}=63$ & $f_{\mathrm{in}_{22}}=126$ & $f_{\mathrm{in}_{23}}=252$ \\
\hline$\hat{f}_{\mathrm{in}_{3}} / f_{s} \equiv 1 / 8$ & $N_{p_{3}}=31$ & $f_{\mathrm{in}_{31}}=31$ & $f_{\mathrm{in}_{32}}=62$ & $f_{\mathrm{in}_{33}}=124$ \\
\hline
\end{tabular}

* All frequencies are in $\mathrm{MHz}$ or MS/s.

The reconfigurable BIST architecture shown in Fig. 2 can be efficiently integrated (e.g. with small area overhead), as detailed explained in [8]. As a result, it is a very attractive solution for functional testing of high speed ADCs.

\section{EXPERIMENTAL RESULTS}

We will consider two extreme evaluation modes:

i) $\mathrm{ADC}$ driven by the external analog input and clock (this mode assesses the standalone ADC performance); ii) ADC stimulated with the internal analog input and clock (this mode assesses the performance of the whole BIST system, i.e., ADC + PLLCK + PLLIN).

The test modes above are evaluated using the PCB board (with a wire-bonded die) shown in Fig. 3. The evaluation mode where both the analog input and clock are generated externally is the most demanding in terms of test equipment resources.

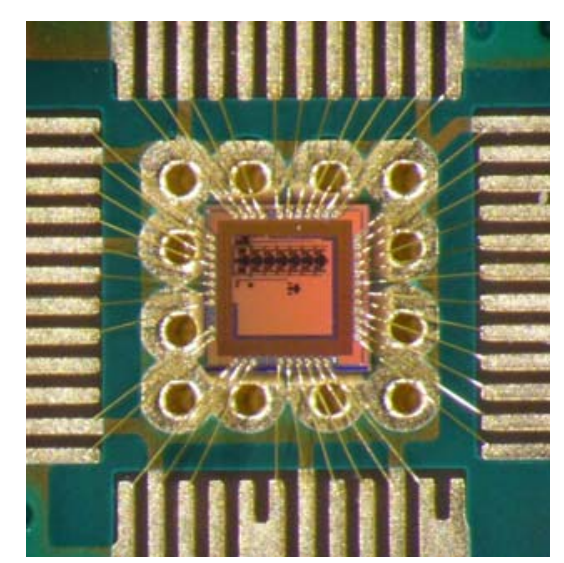

(a)

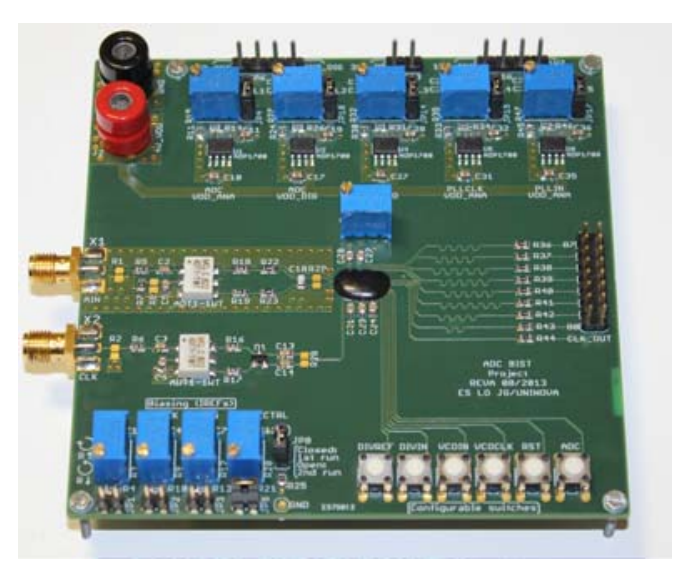

(b) 
Fig. 3. Photograph of a wire-bonded die before glob top encapsulation (a) and of a completely assembled test board (b). Die dimensions are $1525 \times 1525 \mu \mathrm{m}^{2}$. PLLIN core occupies an area of only $138 \mu \mathrm{m} \times 282$ $\mu \mathrm{m}$ and PLLCK occupies $92 \mu \mathrm{m} \times 138 \mu \mathrm{m}$.

The high-quality (i.e., very low phase noise) external analog input is provided by an RF signal generator, Marconi Instruments 2041. In order to remove the harmonics of the generated signal (intrinsic to this kind of generator), the analog input is bandpass or low-pass filtered before being applied to the test board. In this work, the following Mini-Circuits passive filters were used: SBP-10.7, SLP-100+, SLP-250+, SLP550+, and SLP750+. The external clock signal, both for ADC clocking or for the reference clock of the PLLs, is provided by another RF signal generator, Rohde\&Schwarz SMB100A/SMB-B112. This generator also has very low phase noise. For instance, the integrated rms jitter in the bandwidth from $100 \mathrm{~Hz}$ to 2 $\mathrm{GHz}$ at $1 \mathrm{GHz}$ is merely 210 fs. The ADC output data are captured with a logic analyzer, Agilent $16702 \mathrm{~B} / 16715 \mathrm{~A}$, and the data are then remotely transferred to a personal computer and processed in MATLAB. A set of on-board push-button switches allows the configuration of the system and the selection of the desired test mode.

The standalone performance of the ADC, when both the analog input and the clock are provided externally, is presented. Following that, the whole ADC BIST performance is presented, when the analog input and the clock are both generated internally by PLLIN and PLLCK, respectively.

The results are presented in terms of the performance parameters: SINAD, SNR, and THD. Since the ADC topology is based on a two-channel time-interleaving (TI) array, it is also interesting to analyse the spurious tones caused by TI. The THD values presented account up to the $13^{\text {rd }}$ harmonic. The even harmonics are insignificant with respect to the odd ones due to the use of differential signalling and very symmetrical and careful layouts. All results presented are derived from $\underline{512 \text { collected samples, }}$ coherent spectra and no averaging is applied.

\section{DUT with External Analog Input and Clock:}

In this case the converter is stimulated with external analog input and clock. This evaluation gives a reference performance which can then be contrasted with the other evaluation case, in which the analog input and clock are generated on-chip.

The measured SINAD, SNR, THD, and TI spurious components versus input frequency for a randomly selected die sample are shown in Fig 4. With a full-scale input tone, all metrics remain approximately flat up to the Nyquist frequency. 


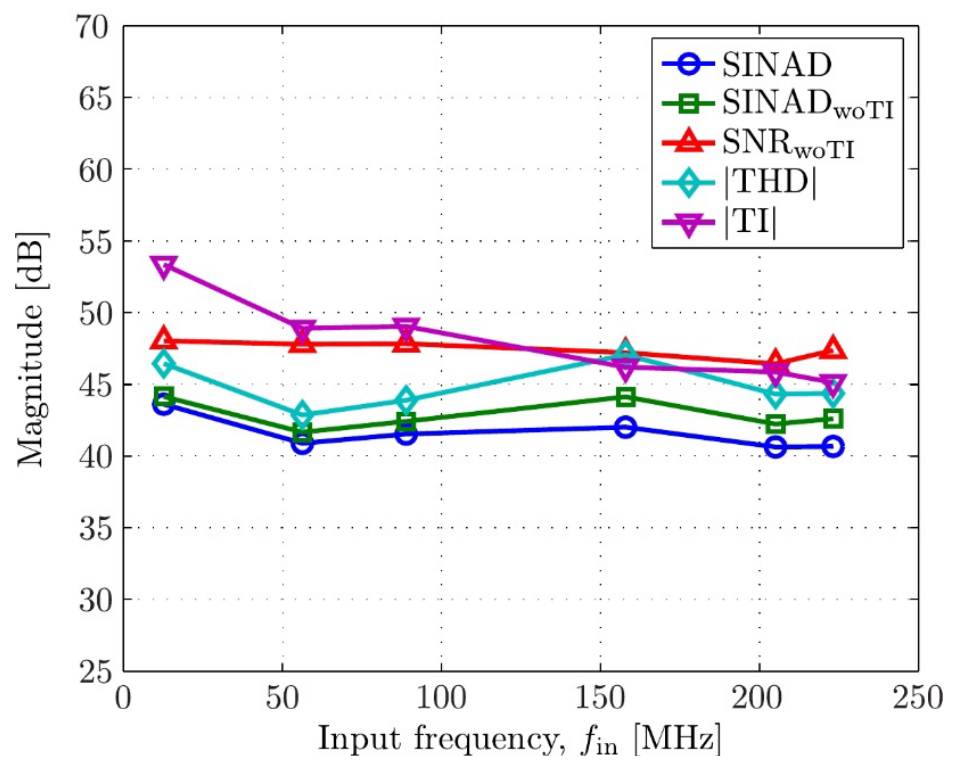

Fig. 4 Measured SINAD, SNR, THD, and TI spur versus $f_{\text {in }}$ at $f_{s} \approx 464 \mathrm{MS} / \mathrm{s}$ and $V_{\text {in }} \approx-0.1 \mathrm{dBFS}$ (timeinterleaving spur is removed, i.e. "woTI").

\section{DUT with Internal Analog Input and Clock}

In this case both the analog input signal and the clock signal are provided internally by means of PLLIN and PLLCK, respectively. Thus, the complete BIST system is evaluated.

The amplitude of the sinusoidal signal generated by PLLIN directly influences the THD of the analog input signal to the $\mathrm{ADC}$, because the voltage-controlled oscillator produces more distortion as the oscillation amplitude increases, and also because the linear buffer that follows the VCO and drives the track-and-hold stage of the ADC becomes more nonlinear as the input signal increases.

Since in this work the distortion of the VCO dominates and increases when the output differential

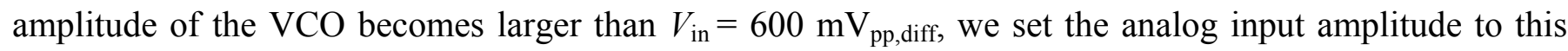

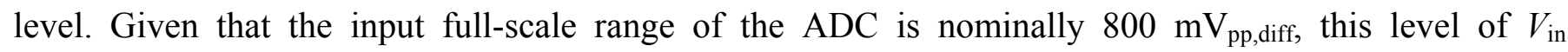
corresponds to $-2.5 \mathrm{dBFS}$. If we were not constrained by the distortion of the VCO, we would choose a larger analog input level, e.g., $-0.1 \mathrm{dBFS}$ (corresponding to $790 \mathrm{mV}_{\text {pp,diff }}$ in this case), in order to exercise almost all output codes of the ADC.

Table $3-\mathrm{H} 3$ and TI spur.

\begin{tabular}{ccc}
\hline Testing case & $\mathrm{H} 3[\mathrm{~dB}]$ & $\mathrm{TI}$ spur $[\mathrm{dB}]$ \\
\hline 1) External input and clock & -48.3 & -44.4 \\
2) Internal input and clock & -41.4 & -42.2 \\
\hline * Obtained from the measured output spectra $(512$ points $)$ \\
of one of the test samples at $f_{s}=464.64 \mathrm{MS} / \mathrm{s}(=$ \\
$256 \times 3.63 / 2 \mathrm{MHz}), f_{\text {in }}=226.875 \mathrm{MHz}(=125 \times$ \\
$3.63 / 2 \mathrm{MHz})$ with $V_{\text {in }} \approx-2.5 \mathrm{dBFS}$ for the two cases.
\end{tabular}


Table 3 summarizes the highest harmonic and TI tone from the measured output spectra for the evaluation cases. For a fair comparison, we use the same frequencies and amplitude for all cases. We note in case 1) that besides the fundamental tone, a set of odd harmonics, dominated by the third harmonic $(-48.3 \mathrm{~dB})$, and the TI spurious tone $(-44.4 \mathrm{~dB})$, which is the strongest in the spectrum.

In case 2), the third harmonic level is $-41.4 \mathrm{~dB}$ and the TI spurious tones is $-42.2 \mathrm{~dB}$. We have a $6.9 \mathrm{~dB}$ difference in the third harmonic and a $2.2 \mathrm{~dB}$ difference in the TI spurious components level. The larger discrepancy in the third harmonic level is mainly due to the increased distortion introduced by the VCO of PLLIN (even though we are using a $V_{\text {in }} \approx-2.5 \mathrm{dBFS}$ ).

Similar conclusions can be drawn analysing the output spectra of other two randomly selected die samples.

In summary, we can say that the quality of the analog input and clock signals produced by the PLLs of the BIST scheme proposed in this work allows us to evaluate an ADC with a SFDR as high as about 41.4 $\mathrm{dB}$ when sampling an almost-Nyquist-frequency tone at about $500 \mathrm{MS} / \mathrm{s}$.

\section{Discussion}

The BIST scheme proposed in this work can be compared with other works in the literature, as shown in Table 4.

One of the key requisites of a BIST circuitry is small area overhead, since the extra silicon area affects both the overall cost and yield of the ICs. As we can see in Table 4, our work has an almost fourfold area improvement compared with the most area-efficient implementation, even ignoring that the other works do not deal with the clock signal generation. This improvement is achieved by a judicious selection of circuit topologies and techniques. For instance, instead of using area-hungry LC oscillators to build up the voltage-controlled oscillators of the phase-locked loops, we use simple RC oscillators. We use a capacitance multiplication scheme to reduce the capacitances, and consequently the silicon area, of the loop filters of the PLLs.

The power dissipation of the BIST circuits is not very important because they are used only during a fraction of the operation time of the remaining (core) circuits, usually during the power-on or during idle time slots. After the self-test procedures, the BIST circuits are turned-off, dropping the power dissipated by these circuits to (almost) zero. Hence, even though the BIST circuits proposed in this work dissipate a relatively high power, $26 \mathrm{~mW}$, this is acceptable for the target application.

We compare the quality of the analog input signal to the DUT using the SFDR metric. Even though the measured SFDR of this work is worse than that of [10]-[12], it is obtained for much higher analog input 
frequencies and amplitudes. For example, the SFDR of $74.1 \mathrm{~dB}$ reported in [10] is for an analog input frequency as low as $10 \mathrm{MHz}$ and for a low differential amplitude, $228 \mathrm{mV}_{\mathrm{pp}}$. In general, it is unfeasible or very difficult to keep a high SFDR with high analog input frequency and/or amplitude, since the circuits suffer from higher distortions or bandwidth. This is shown in the SFDR of $25.3 \mathrm{~dB}$ reported in [9], where the input frequency is $312 \mathrm{MHz}$.

The main limitations of the generated analog input and clock signals of the proposed BIST scheme are the following:

- Distortion in the analog input signal. The sources of these distortions are the nonlinearity of the VCO of PLLIN, and also the nonlinearity of the buffer that follows this VCO and drives the front-end trackand-hold of the ADC.

- Phase noise (or jitter) in the analog input and clock signals. The source of this noise is mainly the noise in the active devices of the VCOs in PLLIN and PLLCK.

- Deterministic reference spurious tones, which are nonidealities (mainly mismatches) in the charge pumps of both PLLCK and PLLIN.

These limitations can be minimized by improving the sizing of the circuits, by enhancements in the circuit topologies, and by careful layouts. Also, some of these limitations are more severe than others. For example, the deterministic reference spurs are not problematic, since they can be easily separated from the actual performance of the ADC under test. In this work, we achieve a worst-case (for three measured samples) SFDR as high as $41.4 \mathrm{~dB}$ for an analog input frequency of about $227 \mathrm{MHz}$ with differential amplitude of $600 \mathrm{mV}_{\mathrm{pp}}$. The SFDR of the generated analog input signal is also dependent on the supply voltage, since reducing this (which is the trend for newer CMOS technologies) equally reduces the voltage headroom available to keep the active devices operating in saturation. In other words, reducing the supply voltage increases the distortion of a given circuit topology, maintaining the same signal swing, unless topology changes (if viable) are made.

The phase noise (or jitter) present in both the output of two PLLs were not measured due to reduced number of $\mathrm{I} / \mathrm{O}$ pins. By post-layout simulations, we can estimate the integrated rms jitter (above $1 \mathrm{MHz}$ offset) of PLLCK as $1.5 \mathrm{ps,} \mathrm{as} \mathrm{indicated} \mathrm{in} \mathrm{Table} \mathrm{4.} \mathrm{This} \mathrm{result,} \mathrm{however,} \mathrm{cannot} \mathrm{be} \mathrm{compared} \mathrm{with} \mathrm{those}$ of other works, since these works only address the analog input signal generation. 
Table 4 - Performance summary and comparison with prior works.

\begin{tabular}{lcccccc}
\hline & & This Work & {$[9]^{*}$} & {$[10]^{*}$} & {$[11]^{*}$} & {$[12]$} \\
\hline Technology & {$[\mu \mathrm{m}]$} & 0.13 & 0.13 & 0.13 & 0.8 & 0.35 \\
Area & {$\left[\mathrm{mm}^{2}\right]$} & 0.052 & $0.51^{\dagger}$ & 0.186 & 0.833 & 0.66 \\
Power & {$[\mathrm{mW}]$} & 26.0 & 39.5 & 4.04 & 36.5 & N/A \\
Supply Voltage & {$[\mathrm{V}]$} & 1.2 & 1.4 & 1.2 & N/A & 3.3 \\
ADC Input Voltage & {$\left[\mathrm{mV}_{\mathrm{pp}}\right]$} & 600 & 149 & 228 & N/A & $-6 \mathrm{dBFS}$ \\
ADC Input Freq. & {$[\mathrm{MHz}]$} & 227 & 312 & 10 & 40 & 0.017 \\
SFDR & {$[\mathrm{dB}]$} & $41.4^{\S}$ & 25.3 & 74.1 & $65.4^{\ddagger}$ & $>76$ \\
ADC Clock Freq. & {$[\mathrm{MHz}]$} & 465 & N/A & N/A & N/A & 6.144 \\
ADC Clock Jitter & {$[\mathrm{ps}]$} & $1.5^{* *}$ & N/A & N/A & N/A & N/A \\
\hline
\end{tabular}

* Do not deal with the clock signal generation.

$\dagger$ Area of the read-only memory not included.

$\ddagger$ SFDR measured at $1 \mathrm{MHz}$ frequency.

$\S$ Ignoring PLLs reference spurs.

** Jitter rms integrated above $1 \mathrm{MHz}$ and estimated from simulation.

\section{CONCLUSIONS}

In this paper we present an overview of challenges in testing ADCs at high frequencies, which will be a hot topic in near future, due to the continuous advances of CMOS technologies. We review the advantages and disadvantages of BIST approaches and show that BIST can be a promising solution for functional and dynamic testing of high speed ADCs.

We present a fully integrated BIST technique, together with experimental results, targeting highfrequency and moderate resolution ADCs. This fully integrated, low cost, and reconfigurable architecture for coherent self-testing of high speed ADCs is based on two synchronized PLLs, one to synthesize the analog input signal and another to synthesize the clock signal for the ADC under test. This synchronization is a key feature of the proposed approach, since it allows coherent sampling. With coherent sampling, the digital output data of the converter are well-behaved and their spectrum is unambiguous, since it is not necessary to worry with spectral leakage, windowing, etc. Hence, with a reduced record length and a relatively low-complexity DSP hardware, it is feasible to do a reliable spectral analysis to assess the dynamic performance of the ADC.

Even though the proof-of-concept is made with a moderate resolution converter, the method proposed can be extended to low- and high-resolution ADCs. The proposed BIST architecture for ADCs is reconfigurable: it is possible to synthesize different analog input and clock frequencies by simply changing 
the frequency dividers values related to the PLLs. The frequency ranges depend on the locking ranges of the PLLs. The complete BIST system has been implemented in a $0.13 \mu \mathrm{m}$ CMOS technology and experimentally evaluated to validate the proposed method.

\section{REFERENCES}

[1] P. Daponte, L. D. Vito, S. Rapuano and I. Tudosa, "Analog-to-information converters in the wideband rf measurement for aerospace applications: current situation and perspectives," in IEEE Instrumentation \& Measurement Magazine, vol. 20, no. 1, pp. 20-28, February 2017.

[2] J. Blair, "Histogram measurement of ADC nonlinearities using sine waves," IEEE Trans. Instrum. Meas., vol. 43, pp. 373383, Mar. 1994.

[3] J. Blair, "Selecting test frequencies for sinewave tests of ADCs," in Proc. IEEE Instrumentation and Measurement Technology Conference (IMTC'02), 2002, pp. 189-193.

[4] T. Sundstrom, C. Svensson, and A. Alvandpour, "A 2.4 GS/s, singlechannel, $31.3 \mathrm{~dB}$ SNDR at Nyquist, pipeline ADC in 65 nm CMOS,” IEEE J. Solid-State Circuits, vol. 46, pp. 1575-1584, Jul. 2011.

[5] M. L. Bushnell and V. D. Agrawal, Essentials of Electronic Testing for Digital, Memory and Mixed-Signal VLSI Circuits. New York: Kluwer Academic Publishers, 2002.

[6] Future Fab International, Special Focus: International Technology Roadmap for Semiconductors, vol. 36, Jan. 2011.

[7] E. Santin, L. B. Oliveira, B. Nowacki, and J. Goes, "A fully integrated and reconfigurable architecture for coherent selftesting of high speed analog-to-digital converters," IEEE Trans. Circuits Syst. I, vol. 58, pp. 1531-1541, Jul. 2011.

[8] E. Santin, “A Built-In Self-Test Technique for High Speed Analog-to-Digital Converters,” Ph.D. dissertation, Universidade Nova de Lisboa, Lisbon, 2014. Accessed on: August 5, 2019. [Online]. Available: http://hdl.handle.net/10362/14389

[9] J. Wibbenmeyer and C.-I. H. Chen, "Built-in self-test for low-voltage high-speed analog-to-digital converters," IEEE Trans. Instrum. Meas., vol. 56, pp. 2748-2756, Dec. 2007.

[10] M. M. Elsayed and E. Sanchez-Sinencio, “A low THD, low power, high' output-swing time-mode-based tunable oscillator via digital harmoniccancellation technique," IEEE J. Solid-State Circuits, vol. 45, pp. 1061- 1071, May 2010.

[11] B. Dufort and G. W. Roberts, “On-chip analog signal generation for mixed-signal built-in self-test,” IEEE J. Solid-State Circuits, vol. 34, pp.

318-330, Mar. 1999.

[12] H. C. Hong, F. Y. Su, and S. F. Hung, "A fully integrated built-in self-test ADC based on the modified controlled sinewave fitting procedure," IEEE Transactions on Instrumentation and Measurement, vol. 59, no. 9, pp. 2334-2344, Sept 2010 .

Edinei Santin (S'07-M'15) was born in Marau, Brazil, in 1986. He received the BSc degree in Electrical Engineering from Federal University of Santa Maria, Santa Maria, Brazil, in 2008, and the PhD degree in Electrical and Computer Engineering from Universidade Nova de Lisboa, Lisbon, Portugal, in 2014. From March 2015 to February 2018 he was a Senior Fellow at the European Organization for Nuclear Research, Geneva, Switzerland, working on the design of hybrid and monolithic silicon pixel detectors for medical imaging and future particle colliders. His research interests include analog and mixed-signal integrated circuits, data converters, monolithic and hybrid silicon pixel detectors, and built-in self-testing and self-calibration techniques.

Luis B. Oliveira was born in Lisbon, Portugal, in 1979. He graduated with a degree in electrical and computer engineering, and a Ph.D. degree, in 2002 and 2007 respectively, from Instituto Superior Técnico (IST), Technical University of Lisbon. In 2007, he joined the teaching staff of the Department of Electrical Engineering of Faculdade de Ciências e Tecnologia, Universidade Nova de Lisboa, and is currently a researcher at CTS-UNINOVA.

Prof. Oliveira is an IEEE Senior Member, vice-chair of the IEEE Portugal Executive Committee, for the period 2018-2019, and chair of the Broadcast Technology Society, Circuits and Systems Society, and Consumer Electronics Society, IEEE Joint Chapter, since 2016. He is a member of the IEEE CASS Analog Signal Processing Technical Committee, ASPTC, (the largest within CASS), since 2008. He has more than 100 publications in International Journals and Conferences, and is co-author of two books: "Analysis and Design of Quadrature Oscillators" (Springer, 2008) and "Wideband CMOS Receivers", (Springer, 2015). He is currently an Associate Editor of the IEEE Transactions on Circuits and Systems - II, TCAS-II, for the term 20182019.

Joao Goes received the B.Sc., M.Sc., and Ph.D. degrees in electrical and computer engineering from Instituto Superior Tecnico (IST), Technical University of Lisbon, Lisbon, Portugal, in 1992, 1996, and 2000, respectively, and the Agregado degree (Habilitation degree) in electronics from the Universidade Nova de Lisboa (UNL), Caparica, Portugal, in 2012. Since April 1998, he has been with the Department of Electrical Engineering (DEE), Faculdade de Cincias e Tecnologia (FCT), UNL, 
where he is currently a Full Professor and has been the Department Head since July 2012. Since 1998, he has been a Senior Researcher, responsible for the Micro/Nanoelectronics Research Unit, with the Centre of Technology and Systems (CTS), Instituto de Desenvolvimento de Novas Tecnologias (UNINOVA). In November 2012, he also became the Director of CTS.

Since 1992, he has participated and led several national and European projects in science, technology, and training. He has also supervised (graduated) $12 \mathrm{Ph} . \mathrm{D}$. theses, $24 \mathrm{M}$.Sc. theses, and nine graduation projects. He has published over 180 papers in international journals and leading IEEE conferences. He was the TPC Co- Chairman of IEEE ISCAS 2015 held in Lisbon on May, 2015 and the TPC Co-Chairman of PRIME 2016. He is currently an Associate Editor of the IEEE TRANSACTIONS ON CIRCUITS AND SYSTEMSII for the period 2016-2019. 
Approach

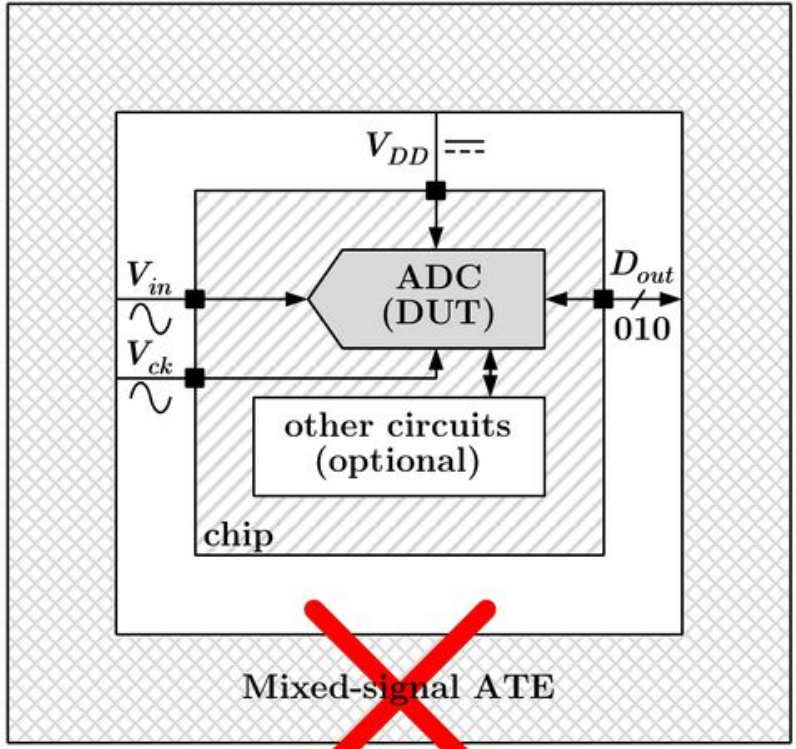

Proposed

Approach

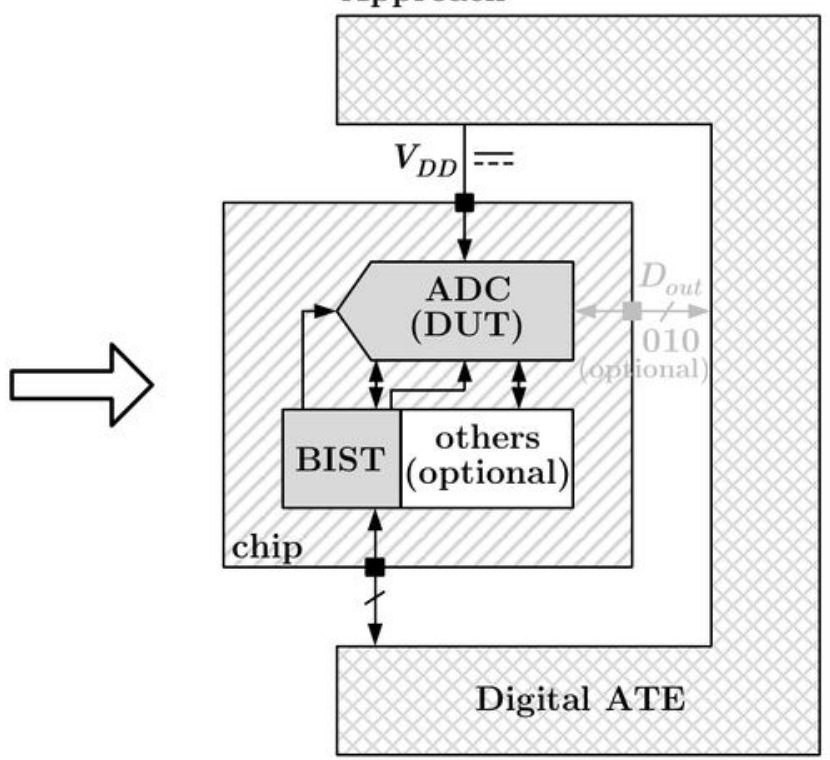




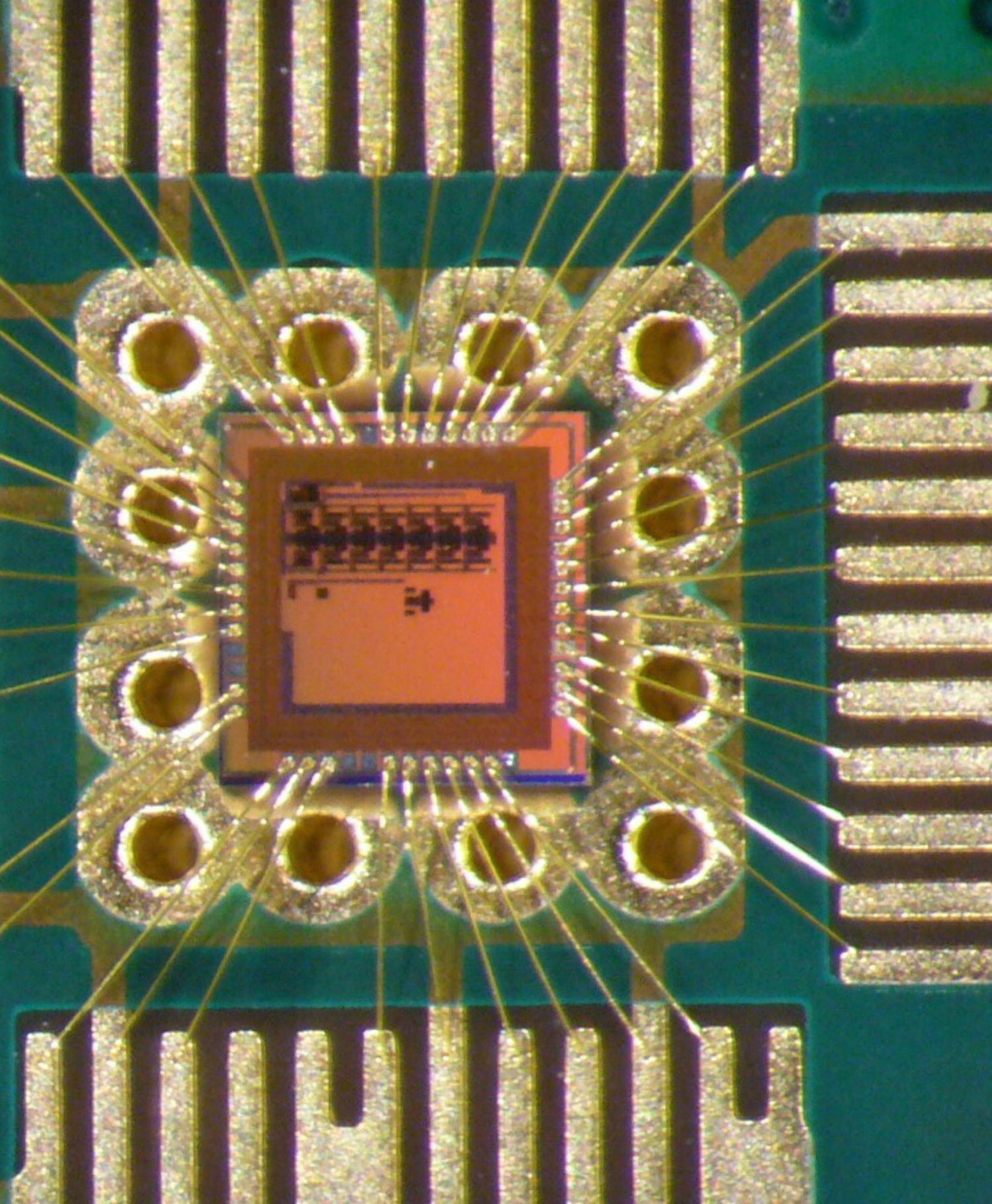









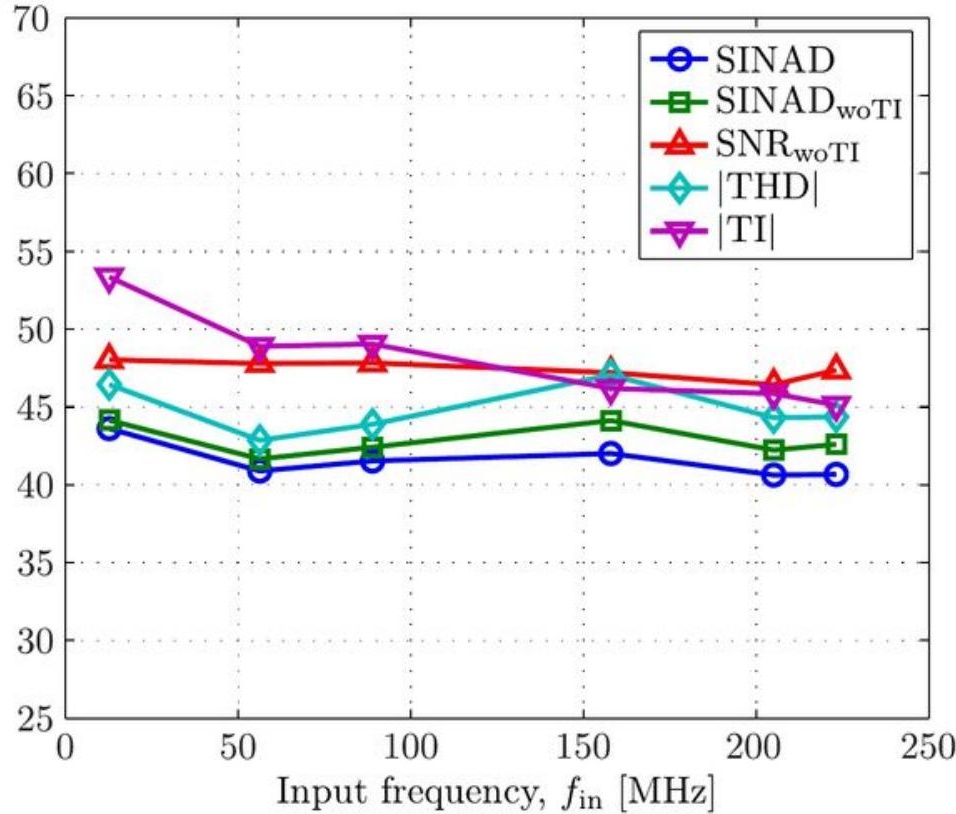




\begin{tabular}{|c|c|}
\hline vertical testability (wafer to system) & area overhead \\
\hline high diagnosis resolution & performance penalties \\
\hline full-speed testing & additional design time \& effort \\
\hline reduced need for external test equipment & additional risk to project \\
\hline reduced test development time \& effort & difficulty to reach full accuracy \\
\hline reduced manufacturing test time \& effort & \\
\hline reduced time-to-market & \\
\hline
\end{tabular}




\begin{tabular}{|l|l|lll|}
\hline \multicolumn{2}{|l}{$f_{\mathrm{ref}} \equiv 4 \mathrm{MHz}$} & \multicolumn{1}{|c}{$\hat{f}_{s_{1}} \equiv 250$} & $\hat{f}_{s_{2}} \equiv 500$ & $\hat{f}_{s_{3}} \equiv 1000$ \\
\hline \multirow{2}{*}{$N \equiv 6$ bits } & \multirow{2}{*}{$N_{s}=256$} & $M_{1}=4$ & $M_{2}=2$ & $M_{3}=1$ \\
\cline { 3 - 5 } & & $f_{s_{1}}=256$ & $f_{s_{2}}=512$ & $f_{s_{3}}=1024$ \\
\hline$\hat{f}_{\mathrm{in}_{1}} / f_{s} \equiv 1 / 2$ & $N_{p_{1}}=127$ & $f_{\mathrm{in}_{11}}=127$ & $f_{\mathrm{in}_{12}}=254$ & $f_{\mathrm{in}_{13}}=508$ \\
\hline$\hat{f}_{\mathrm{in}_{2}} / f_{s} \equiv 1 / 4$ & $N_{p_{2}}=63$ & $f_{\mathrm{in}_{21}}=63$ & $f_{\mathrm{in}_{22}}=126$ & $f_{\mathrm{in}_{23}}=252$ \\
\hline$\hat{f}_{\mathrm{in}_{3}} / f_{s} \equiv 1 / 8$ & $N_{p_{3}}=31$ & $f_{\mathrm{in}_{31}}=31$ & $f_{\mathrm{in}_{32}}=62$ & $f_{\mathrm{in}_{33}}=124$ \\
\hline
\end{tabular}

* All frequencies are in $\mathrm{MHz}$ or MS/s. 
1) External input and clock

2) Internal input and clock
$-48.3$

$-41.4$
$-44.4$

$-42.2$

* Obtained from the measured output spectra (512 points) of one of the test samples at $f_{s}=464.64 \mathrm{MS} / \mathrm{s}(=$ $256 \times 3.63 / 2 \mathrm{MHz}), f_{\text {in }}=226.875 \mathrm{MHz}(=125 \times$ $3.63 / 2 \mathrm{MHz})$ with $V_{\text {in }} \approx-2.5 \mathrm{dBFS}$ for the two cases. 


\section{$[9]^{*} \quad[10]^{*}$}

$[11]^{*}$

[12]

\section{Technology}

Area

Power

Supply Voltage

ADC Input Voltage

ADC Input Freq.

SFDR

ADC Clock Freq.

ADC Clock Jitter

$[\mu \mathrm{m}]$
$\left[\mathrm{mm}^{2}\right]$
$\left[\mathrm{mW}^{2}\right]$
$[\mathrm{V}]$
$\left[\mathrm{mV}_{\mathrm{pp}}\right]$
$[\mathrm{MHz}]$
$[\mathrm{dB}]$
$[\mathrm{MHz}]$

[ps]
0.13

0.052

26.0

1.2

600

227

$41.4^{\S}$

465

$1.5 * *$
0.13

$0.51^{\dagger}$

39.5

1.4

149

312

25.3

N/A

N/A
0.13

0.186

4.04

1.2

228

10

74.1

N/A

N/A
0.8

0.833

36.5

N/A

N/A

40

$65.4^{\ddagger}$

N/A

N/A
0.35

0.66

N/A

3.3

$-6 \mathrm{dBFS}$

0.017

$>76$

6.144

N/A

* Do not deal with the clock signal generation.

$\dagger$ Area of the read-only memory not included.

$\ddagger$ SFDR measured at $1 \mathrm{MHz}$ frequency.

$\S$ Ignoring PLLs reference spurs.

** Jitter rms integrated above $1 \mathrm{MHz}$ and estimated from simulation. 